\title{
Increased Proportion of Hematopoietic Stem and Progenitor Cell Population in Cord Blood of Neonates Born to Mothers with Gestational Diabetes Mellitus
}

\author{
Orsolya Hadarits, ${ }^{1, *}$ András Zóka, ${ }^{2, *}$ Gábor Barna, ${ }^{3}$ Zahra Al-Aissa, ${ }^{2}$ Klára Rosta, ${ }^{1,4}$ János Rigó Jr., \\ Alexandra Kautzky-Willer, ${ }^{5}$ Anikó Somogyi, ${ }^{2}$ and Gábor Firneisz ${ }^{2,6}$
}

We assessed the hematopoietic stem and progenitor cell (HSPC) population in the cord blood of neonates born to mothers with gestational diabetes mellitus (GDM) in a hypothesis generating pilot study, due to that, neonatal polycythemia may be the consequence of GDM pregnancy. Forty-five pregnant women with GDM (last trimester mean $\mathrm{HbA} 1 \mathrm{C}=33.9 \mathrm{mmol} / \mathrm{mol}$ ) and 42 (nondiabetic) control pregnant women were enrolled after their routine $75 \mathrm{~g}$ oral glucose tolerance test (OGTT) between the 24th and 28th gestational week (with expected differences in their mean routine clinical characteristics: plasma glucose at OGTT: $0^{\prime}=5.07$ vs. $4.62 \mathrm{mM}, 120^{\prime}=8.9$ vs. $5.76 \mathrm{mM}$, age $=35.07$ vs. 31.66 years, prepregnancy body mass index $=27.9$ vs. $23.9 \mathrm{~kg} / \mathrm{m}^{2}, \mathrm{GDM}$ vs. control, respectively) on a voluntary basis after signing the informed consent. EDTA-treated cord blood samples were analyzed by flow cytometry and the software Kaluza1.2 using CD45 and CD34-specific fluorescent antibodies to identify the HSPC population (CD34 ${ }^{+}$cells within the $\mathrm{CD} 45^{\mathrm{dim}}$ blast gate). The proportion of $\mathrm{CD} 34^{+} \mathrm{CD} 45^{\mathrm{dim}} \mathrm{HSPC}$ among the nucleated cells was significantly $(P<0.05$, statistical power $=60.8 \%$ ) higher in the cord blood samples of neonates born to mothers with GDM (median $0.38 \%)$ compared to neonates born to nondiabetic mothers (median $0.32 \%)$ and according to treatment types $(P<0.05)$ median: control $0.32 \%$, GDM-diet only $0.37 \%$, GDM-on insulin $0.45 \%$; control versus GDM on insulin $(P<0.05)$. The increased proportion of circulating $\mathrm{CD} 34^{+} \mathrm{CD} 45^{\mathrm{dim}}$ cells in the cord blood may possibly be related to altered fetal stem cell mobilization in GDM pregnancy, yet these results should be interpreted only as preliminary due to the small sample sizes.

\section{Introduction}

$\mathrm{D}$ ESPITE NEONATAL POLYCYTHEMIA being more common in infants born to mothers with gestational diabetes mellitus (GDM) than in infants in the general population-to our knowledge — no published study has assessed the hematopoietic stem and progenitor cell (HSPC) population in the cord blood of neonates born to mothers with GDM.

Even in infants born to women with only diet-controlled GDM, neonatal hematocrit values above $60 \%$ are occurring at a higher than $10 \%$ rate [1], and the prevalence of neonatal polycythemia in insulin-requiring GDM and type 1 diabetic pregnancies may even exceed the $30 \%$ and $40 \%$, respectively $[2,3]$. The pathophysiology of this neonatal complication of maternal diabetes has been studied focusing on later stages of hematopoiesis. Authors suggested decades ago that the increased human umbilical plasma erythropoietin (Epo) levels were secondary due to fetal hyperinsulinemia [4]; however, no data were available about the cord hematopoietic stem cell (HSC) population in GDM before our study.

In addition, the DPP-4-CXCL12 axis has been recently identified as an important regulator of HSC mobilization in the adult human bone marrow in response to ischemic stimuli [5,6]. We recently reported that the serum DPP-4 enzymatic activity was decreased in the cord blood of neonates born to mothers with GDM [7], and therefore, it could be hypothesized that the cord HSC/HSPC population may be altered in GDM.

\section{Materials and Methods}

\section{Research design}

This study has been conducted in a single Hungarian center in the framework of the New Horizons Initiative of the

\footnotetext{
${ }^{1}$ 1st Department of Obstetrics and Gynecology, ${ }^{2}$ 2nd Department of Internal Medicine, and ${ }^{3} 1$ st Department of Pathology and Experimental Cancer Research, Semmelweis University, Budapest, Hungary.

${ }^{4}$ Department of Obstetrics and Fetomaternal Medicine, University Hospital, Vienna, Austria.

${ }^{5}$ Division of Endocrinology and Metabolism, Department of Medicine 3, Medical University of Vienna, Vienna, Austria.

${ }^{6}$ Hungarian Academy of Sciences, Semmelweis University, Molecular Medicine Research Group, Budapest, Hungary.

*These authors contributed equally to this work.

(C) Orsolya Hadarits et al., 2015; Published by Mary Ann Liebert, Inc. This Open Access article is distributed under the terms of the Creative Commons Attribution Noncommercial License (http://creativecommons.org/licenses/by-nc/4.0/) which permits any noncommercial use, distribution, and reproduction in any medium, provided the original author(s) and the source are credited.
} 
EFSD after receiving approval from the appropriate ethical bodies. Altogether, 90 pregnant women signed the informed consent following the $75 \mathrm{~g}$ oral glucose tolerance test (OGTT) from the 24th to 28th gestational week: cord blood sampling at delivery with HSPC population assessment was possible from umbilical cord blood samples of neonates born to mothers with GDM $(n=45)$ and nondiabetic controls $(n=42)$, and three individuals were not included in the final analysis due to damaged samples.

\section{Patients and cord blood collection}

Altogether, 45 pregnant women with GDM as well as 42 control pregnant women were enrolled immediately after their routine OGTT between the 24th and 28th gestational week on a voluntary basis after signing the informed consent. We designated a control group as individuals who carry a pregnancy and were neither diagnosed with GDM nor with overt diabetes between the 24th and 28th gestational week at OGTT or later throughout the course of their pregnancy. The diagnosis of GDM has been established according to the Hungarian national recommendations (modified 1999 WHO recommendation-GDM: $75 \mathrm{~g}$ OGTT at 24-28 gw: FPG $\geq 6.1 \mathrm{mM}, 120^{\prime} \mathrm{PG} \geq 7.8 \mathrm{mM}$ ). Individuals diagnosed with overt diabetes (classified as diabetes in the pregnancy category in 2013, WHO recommendation, that is, FPG $\geq 7.0 \mathrm{mM}$, $120^{\prime}$ PG $\geq 11.1 \mathrm{mM}$ ) were excluded from the analysis. Cord blood samples were collected from the umbilical vein using the cord clamping technique as soon as it was possible after birth. The most important clinical maternal data were recorded (including the age at delivery, $75 \mathrm{~g}$ OGTT $0^{\prime}$ and 120' min values between the 24th and 28th gestational week, prepregnancy body mass index (BMI), weight gain during pregnancy, and third trimester HbAlc value in the GDM group). The birth weight percentiles were assessed using a downloaded program on fetal weight equations as a published global reference for birth weight percentiles using 3,340 and $3,500 \mathrm{~g}$ as the Hungarian population mean birth weight values at 40 weeks for female and male neonates, respectively [8].

\section{Flow cytometric analysis}

EDTA-treated cord blood samples were analyzed by flow cytometry (Beckman Coulter Navios flow cytometer, Kaluza 1.2 software). Leukocytes were gated and differentiated from debris according to their forward and side scatter. BD
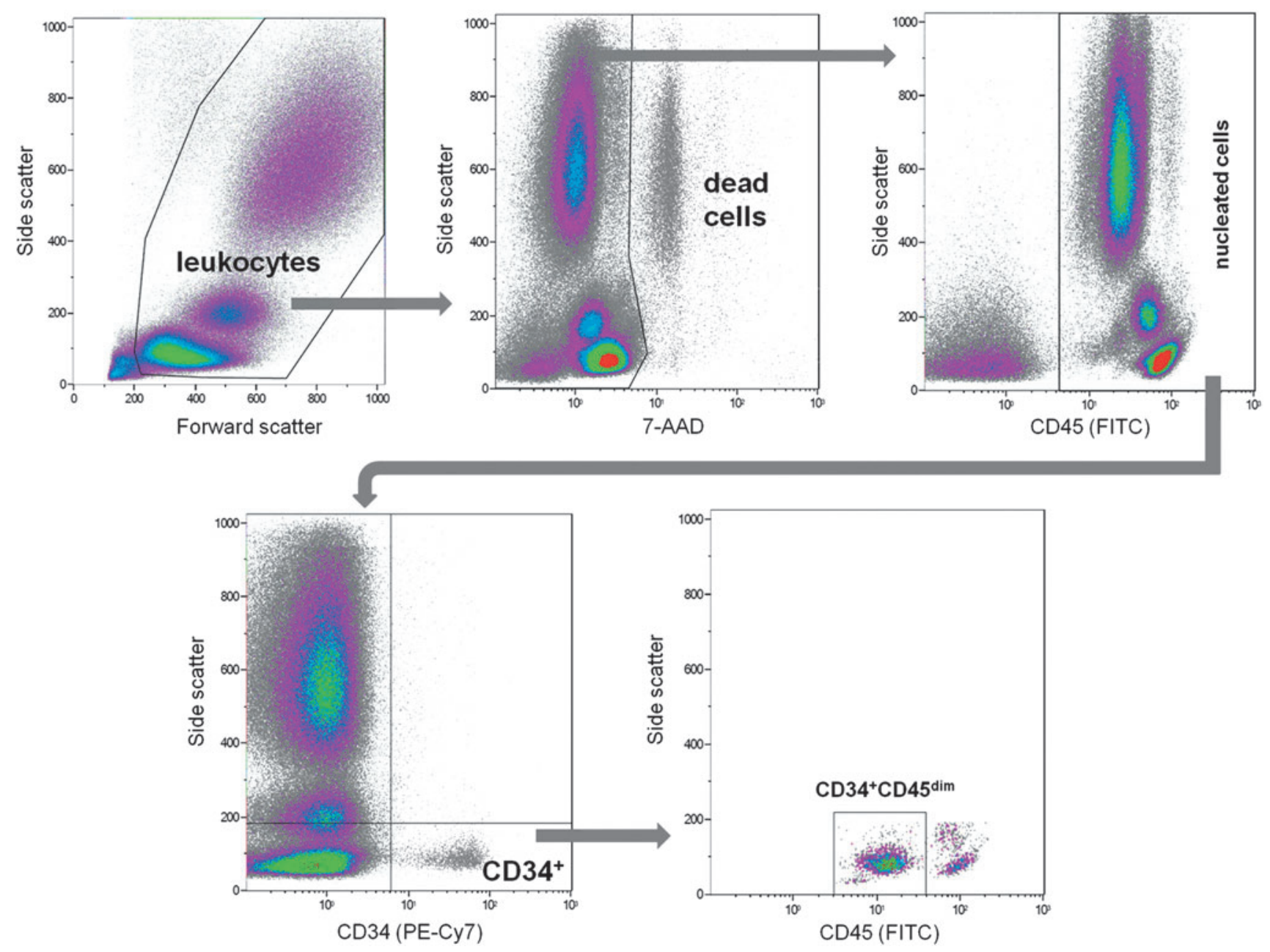

FIG. 1. Leukocytes were separated from debris according to their forward and side scatter. Red blood cells were separated from nucleated cells according to their absent CD45 expression in the undead (nonpermeable for 7-AAD) cell population. The stem and precursor cells were gated within all nucleated cells according to their CD34 expression and their intermediate CD45 expression (CD45 $\left.{ }^{\mathrm{dim}}\right)$. 
Table 1. Clinical Characteristics of the Pregnant Populations Studied

\begin{tabular}{|c|c|c|c|c|c|c|}
\hline \multirow[b]{3}{*}{ Study group } & $\begin{array}{r}\text { Plasm } \\
\text { value } \\
\text { OGTT }(24 \\
\end{array}$ & $\begin{array}{l}\text { glucose } \\
\text { at } 75 \mathrm{~g} \\
28 \mathrm{gw}, \mathrm{mM})\end{array}$ & \multirow{3}{*}{$\begin{array}{c}\text { Prepregnancy } \\
\text { BMI }\left(\mathrm{kg} / \mathrm{m}^{2}\right)\end{array}$} & \multirow{3}{*}{$\begin{array}{c}\text { Age at } \\
\text { delivery } \\
\text { (years) }\end{array}$} & \multirow{3}{*}{$\begin{array}{c}\begin{array}{c}\text { Weight gain } \\
\text { during pregnancy } \\
(\mathrm{kg})\end{array} \\
\text { Mean } \\
(95 \% \mathrm{CI})\end{array}$} & \multirow{3}{*}{ 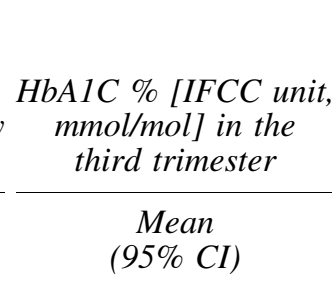 } \\
\hline & \multicolumn{2}{|c|}{ Mean $(95 \%$ CI $)$} & & & & \\
\hline & $O^{\prime}$ & $120^{\prime}$ & & & & \\
\hline GDM & $5.07(4.85-5.29)$ & 8.9 & $\begin{array}{c}27.9 \\
(25.55-30.16)\end{array}$ & $\begin{array}{c}35.07 \\
(33.69-36.43)\end{array}$ & $\begin{array}{c}7.58 \\
(5.43-9.72)\end{array}$ & $\begin{array}{c}5.24(5.08-5.4) \\
{[33.9} \\
(32.15-35.64)]\end{array}$ \\
\hline $\begin{array}{l}\text { Control }(n=42) \\
P \text { value }\end{array}$ & $\begin{array}{c}4.62(4.42-4.83) \\
0.0036\end{array}$ & $\begin{array}{c}5.76(5.41-6.11) \\
<0.0001\end{array}$ & $\begin{array}{c}23.9 \\
(22.74-25.01) \\
0.0024\end{array}$ & $\begin{array}{c}31.66 \\
(29.9-33.42) \\
0.0025\end{array}$ & $\begin{array}{c}14.16 \\
(12.62-15.69) \\
<0.0001\end{array}$ & NA \\
\hline
\end{tabular}

BMI, body mass index; 95\% CI, 95\% confidence interval; GDM, gestational diabetes mellitus; OGTT, oral glucose tolerance test.

Via-Probe (BD Biosciences, San Jose, CA) cell viability solution (7-AAD labeled) was used to differentiate living from dead cells. Cell surface CD45 (label: FITC, isotype: IgG1, $\kappa$, Clone: HI30) and CD34 (label: PE-Cy7, isotype: IgG1, $\kappa$, Clone: 581) were stained with specific fluorescent antibodies (BioLegend, San Diego, CA). Residual red blood cells $(\mathrm{RBC})$ were filtered out from the leukocytes according to their absent CD45 expression.

\section{Identification of stem and progenitor cell populations}

We identified CD34 ${ }^{+}$cells within the gate of the nucleated cells. Circulating HSPCs were defined according to the International Society of Hematotherapy and Graft Engineering (ISHAGE) criteria as CD $34^{+}$HPCs within the $\mathrm{CD} 45^{\mathrm{dim}}$ blast gate, indicating that they express CD45 at a lower intensity than matured leukocytes $[9,10]$. Our gating strategy is indicated on Fig. 1.

\section{Statistical analysis}

Statistica software (version 11; StatSoft, Tulsa, OK) was used for statistical analysis. Kolmogorov-Smirnov test was used to assess normality. As data distributions were nonnormal, the Mann-Whitney $U$ test (MWU) was used to compare the data of the healthy control and GDM study populations on the primary outcomes (proportions of hematopoietic stem and precursor cells). The Kruskal-Wallis ANOVA test was used to compare multiple samples. Student's $t$-test was used to compare means of clinical data (Table 1) due to the normal demographic data distributions in the GDM and control populations according to the Kolmogorov-Smirnov normality test. For paired comparisons, we also performed power analysis using the mean values, the sample size numbers, and the population sigma values.

\section{Results}

\section{Clinical data}

Prepregnancy BMI and the age at delivery were significantly higher in the GDM group compared to the controls. The most important clinical data of the pregnant population are summarized in Table 1. Neonatal birth weight categories according to gestational age and the neonatal gender distributions in the study groups are indicated in Table 2.

\section{Proportions of hematopoietic stem and precursor cells}

The proportion of $\mathrm{CD} 34^{+} \mathrm{CD} 45^{\mathrm{dim}}$ cells (HSPCs) among the nucleated cells was significantly (MWU $P<0.05$, statistical power $=60.8 \%)$ higher in the cord blood samples of neonates born to mothers with GDM (median $0.38 \%$ [SD ${ }^{2}$ 0.07]) compared to neonates born to nondiabetic mothers $\left(0.32 \%\left[\mathrm{SD}^{2} 0.03\right]\right)$. Effect size $(=0.497)$ calculation was also performed according to Cohen.

We found a significant difference (Kruskal-Wallis test $P<0.05)$ in the proportion of HSPCs among the nucleated cord blood cell population among different study groups according to the maternal GDM diagnosis and treatment types (control: median $0.32 \%$ [ $\mathrm{SD}^{2} 0.03$ ], GDM requiring only diet $0.37 \%$ [SD $\left.{ }^{2} 0.07\right]$, and GDM on insulin therapy $0.45 \%$ [SD ${ }^{2}$ 0.05]) (Fig. 2). The Newman-Keuls post hoc test also demonstrated a significant difference between the control and the GDM on insulin study groups $(P<0.05)$.

Although routine neonatal full blood count, hemoglobin level, and serum bilirubin measurements were not feasible in all participating neonates as per guidelines and due to technical difficulties, we detected only a borderline correlation between neonatal hemoglobin and bilirubin levels.

Table 2. The Number of Neonates with Large, Appropriate, or Small Birth Weight for Gestational Age Born to Mothers WITH GDM OR TO CONTrol Mothers

\begin{tabular}{|c|c|c|}
\hline $\begin{array}{l}\text { Distribution of } \\
\text { neonatal birth weight } \\
\text { percentiles [n }(\%)]\end{array}$ & $G D M$ & Control \\
\hline $\begin{array}{l}\text { Entire neonatal study } \\
\text { population (gender distribution) }\end{array}$ & $\begin{array}{l}n=45 \\
(\mathrm{~F}=21 \\
\mathrm{M}=24)\end{array}$ & $\begin{aligned} n & =42 \\
(\mathrm{~F} & =19 \\
\mathrm{M} & =23)\end{aligned}$ \\
\hline$L G A$ & $13(28.9)$ & $9(21.4)$ \\
\hline$A G A$ & $31(68.9)$ & 31 (73.8) \\
\hline$S G A$ & $1(2.2)$ & $2(4.8)$ \\
\hline
\end{tabular}

AGA, appropriate for gestational age; F, female; LGA, large for gestational age; M, male; SGA, small for gestational age. 
FIG. 2. Box plot of the proportion of hematopoietic stem and progenitor cells among nucleated cord blood cells in different study groups according to maternal gestational diabetes mellitus (GDM) diagnosis and insulin therapy.

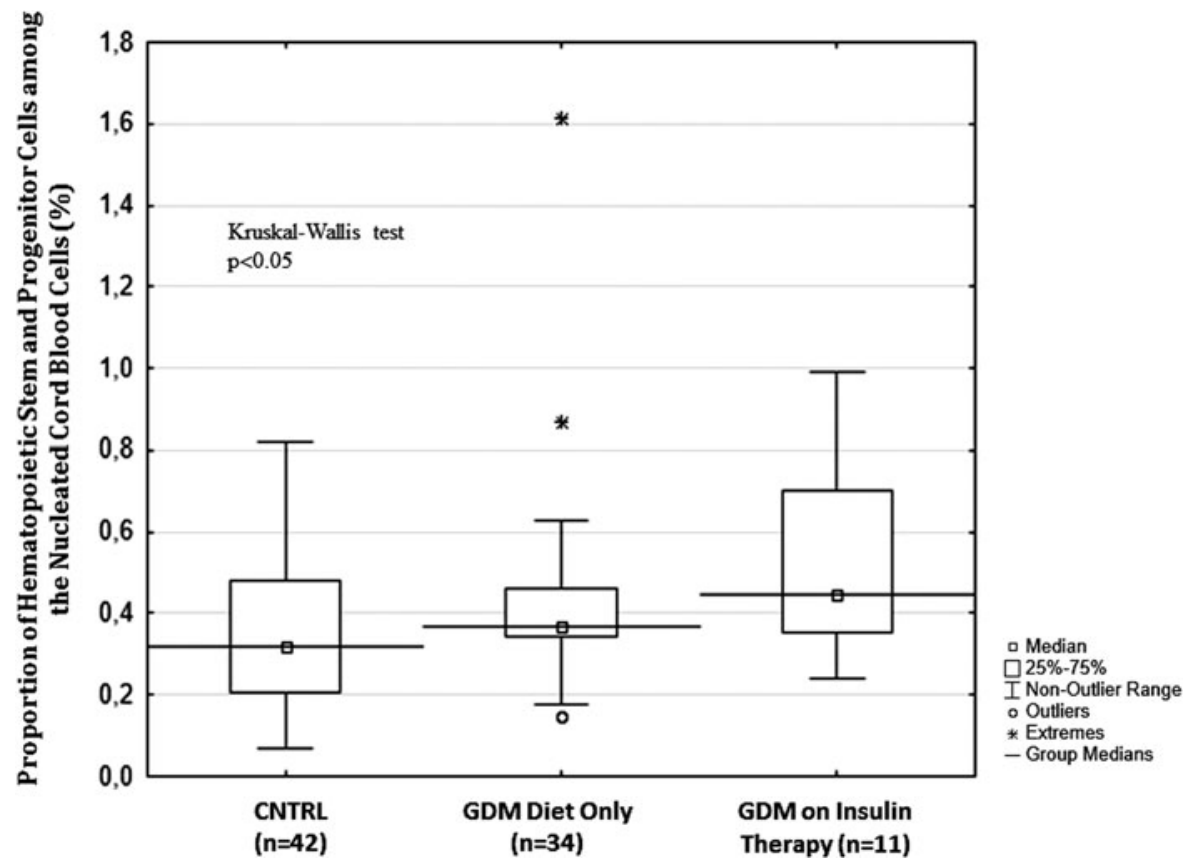

\section{Discussion}

This line of research was motived by major prior clinical findings: a long standing observation from the everyday clinical praxis proved that neonatal polycythemia is a feared complication of diabetic pregnancies occurring with a high enough prevalence to attract clinical attention (over $30 \%$ and $40 \%$ in insulin-requiring GDM or type 1 diabetic pregnancies, respectively). Polycythemia in the infants of diabetic mothers may be associated with serious clinical complications, including the hyperviscosity syndrome, thrombosis, respiratory distress with vascular congestion, heart failure, and cardiomegaly due to increased afterload and neurologic complications, such as stroke.

The $\sim 20 \%-25 \%$ increase in the proportion of circulating HSPCs (CD34 $\left.{ }^{+} \mathrm{CD} 45^{\mathrm{dim}}\right)$ among all nucleated cells in the cord blood of neonates born to mothers with GDM compared to nondiabetic women may be related to the altered fetal stem cell mobilization in GDM pregnancy or possibly due to the insulin therapy.

Neonatal polycythemia was previously explained by the fetal hypoxia-induced increase in Epo production that was closely related to the control of maternal antepartum hyperglycemia (ie, maternal HbA1c levels during the last month of pregnancy in type 1 diabetic women) [11]. Although previously Epo was described to stimulate RBC production downstream in the path when precursor cells already lost their CD34 marker, it has been recently suggested that high systemic levels of Epo may reprogram HSPCs inducing an increased output toward an erythroid fate in vivo [12].

In a prior study, Azouna et al. found that the number of $\mathrm{CD} 4^{+}$cells was higher in the cord blood of neonates with higher birth weight $(>3.5 \mathrm{~kg})$ and born to older mothers [13].

This finding might be in concordance with our findings; due to that, both of the parameters they indentified are clinically associated with GDM, higher maternal age is a risk, and higher birth weight is a possible consequence of GDM. Therefore, our study might provide a plausible common clinical path for their findings (ie, development of GDM); despite that the proportion of neonates in the large for gestational age group was only numerically higher in the GDM group (statistically nonsignificant), the maternal age was significantly higher in the GDM study group.

In addition, a decreased cord serum DPP-4 activity of neonates born to women with GDM compared to the activity in samples of neonates born to nondiabetic women was recently reported [7]. This - theoretically_might implicate an altered cleavage of other chemokines, growth factors, including CXCL12, and may possibly result in an altered mobilization of HSPCs through binding to CXCR4 [14], as this receptor is also present on human CD $34^{+}$cells from the umbilical cord blood [15-17]. Therefore, we may speculate that the DPP-4-CXCL12-CXCR4 axis could also be involved in linking the fetal intrauterine metabolic environment and the stem cell population distributions to certain neonatal complications of the GDM pregnancy, possibly including but not limited to the neonatal polycythemia.

\section{Limitations}

The results presented here could not gain the $80 \%$ statistical power with the limited number of enrolled individuals and the calculated effect size of 0.5 , considered as a "medium" relative size also indicates that the sample sizes should be further expanded and the current results may therefore only be interpreted as preliminary [18]. This was a hypothesis generating pilot study; therefore, no prior sample size calculation was performed. Although our results are coherent with the current knowledge about the regulatory mechanisms involved in HSPC mobilization, due to the limited sample sizes and the distribution of data we may not entirely exclude the occurrence of significances by chance alone. 
The reported data only include cord stem/progenitor cell proportions, and due to the unknown total cell counts, these results represent only one aspect of cell populations that is also characterized by absolute cell numbers. The assessment of more matured precursor forms (ie, the progenitor cell population) with additional markers (eg, when cells already lack CD133 positivity) is currently missing, yet the results are expected to be more pronounced in the view that those progenitor cells are closer to the matured $\mathrm{RBC}$ that are proven to be different in amount in the neonates born to mothers with GDM.

To assess stem cell mobilization in a dynamic manner, both the DPP-4 activity and also the CXCL12 levels should have been measured at tissue levels (eg, bone marrow, placenta, and in the cord blood) to provide evidence for the altered CXCL12 gradient between the bone marrow and the periphery. In our study, we could not present any data about the potential alteration of the CXCL12 gradient between the stem cell niche of the neonatal bone marrow and the periphery; therefore, the discussion in this regard is hypothetical.

Since in a prior report [13] higher $\mathrm{CD} 34^{+}$cell counts were found in the cord blood of neonates born to mothers with higher age, maternal age cannot be excluded as a confounding factor in this analysis.

\section{Acknowledgment}

This study has been funded by the European Foundation for the Study of Diabetes (EFSD) New Horizons Initiative.

\section{Author Disclosure Statement}

No competing financial interests exist.

\section{References}

1. Sarkar S, J Watman, WM Seigel and HA Schaeffer. (2003). A prospective controlled study of neonatal morbidities in infants born at 36 weeks or more gestation to women with diet-controlled gestational diabetes (GDM-class Al). J Perinatol 23:223-228.

2. Hay WW, Jr. (2012). Care of the infant of the diabetic mother. Curr Diab Rep 12:4-15.

3. Lessaris KJ. (2015). Polycythemia of the newborn. In: Rosenkrantz T, ed., http://emedicine.medscape.com/article/ 976319-overview\#a0199.

4. Widness JA, JB Susa, JF Garcia, DB Singer, P Sehgal, W Oh, R Schwartz and HC Schwartz. (1981). Increased erythropoiesis and elevated erythropoietin in infants born to diabetic mothers and in hyperinsulinemic rhesus fetuses. J Clin Invest 67:637-642.

5. DiPersio JF. (2011). Diabetic stem-cell "mobilopathy". N Engl J Med 365:2536-2538.

6. Fadini GP, M Albiero, F Seeger, et al. (2013). Stem cell compartmentalization in diabetes and high cardiovascular risk reveals the role of DPP-4 in diabetic stem cell mobilopathy. Basic Res Cardiol 108:313.

7. Al-Aissa Z, K Rosta, O Hadarits, et al. (2015). Cord serum dipeptidyl-peptidase 4 activity in gestational diabetes. Eur $\mathbf{J}$ Clin Invest 45:196-203.
8. Mikolajczyk RT, J Zhang, AP Betran, JP Souza, R Mori, AM Gulmezoglu and M Merialdi. (2011). A global reference for fetal-weight and birthweight percentiles. Lancet 377:1855-1861.

9. Sutherland DR, L Anderson, M Keeney, R Nayar and I Chin-Yee. (1996). The ISHAGE guidelines for CD34+ cell determination by flow cytometry. International Society of Hematotherapy and Graft Engineering. J Hematother 5: 213-226.

10. Gratama JW, J Kraan, M Keeney, DR Sutherland, V Granger and D Barnett. (2003). Validation of the singleplatform ISHAGE method for CD34(+) hematopoietic stem and progenitor cell enumeration in an international multicenter study. Cytotherapy 5:55-65.

11. Widness JA, KA Teramo, GK Clemons, P Voutilainen, UH Stenman, SM McKinlay and R Schwartz. (1990). Direct relationship of antepartum glucose control and fetal erythropoietin in human type 1 (insulin-dependent) diabetic pregnancy. Diabetologia 33:378-383.

12. Grover A, E Mancini, S Moore, AJ Mead, D Atkinson, KD Rasmussen, D O'Carroll, SE Jacobsen and C Nerlov. (2014). Erythropoietin guides multipotent hematopoietic progenitor cells toward an erythroid fate. J Exp Med 211: $181-188$.

13. Azouna NB, L Berraeis, Z Regaya and F Jenhani. (2011). Immunophenotyping of hematopoietic progenitor cells: comparison between cord blood and adult mobilized blood grafts. World J Stem Cells 3:104-112.

14. Sharma M, F Afrin, N Satija, RP Tripathi and GU Gangenahalli. (2011). Stromal-derived factor-1/CXCR4 signaling: indispensable role in homing and engraftment of hematopoietic stem cells in bone marrow. Stem Cells Dev 20:933-946.

15. Aiuti A, L Turchetto, M Cota, A Cipponi, A Brambilla, C Arcelloni, R Paroni, E Vicenzi, C Bordignon and G Poli. (1999). Human CD34(+) cells express CXCR4 and its ligand stromal cell-derived factor-1. Implications for infection by T-cell tropic human immunodeficiency virus. Blood 94:62-73.

16. Hopman RK and JF DiPersio. (2014). Advances in stem cell mobilization. Blood Rev 28:31-40.

17. Ou X, HA O'Leary and HE Broxmeyer. (2013). Implications of DPP4 modification of proteins that regulate stem/ progenitor and more mature cell types. Blood 122:161-169.

18. Sullivan GM and R Feinn. (2012). Using effect size-or why the $\mathrm{P}$ value is not enough. J Grad Med Educ 4:279-282.

Address correspondence to: Dr. Gábor Firneisz 2nd Department of Internal Medicine Semmelweis University Szentkiralyi Street 46 Budapest $\mathrm{H}-1088$ Hungary

E-mail: firneisz.gabor@med.semmelweis-univ.hu

Received for publication June 11, 2015 Accepted after revision October 22, 2015 Prepublished on Liebert Instant Online October 22, 2015 